\title{
Deciding Networks of Evolutionary Processors
}

\author{
Florin Manea $^{(A)}$ \\ Otto-von-Guericke-Universität Magdeburg, Fakultät für Informatik \\ PSF 4120, D-39016 Magdeburg, Germany \\ manea@iws.cs.uni-magdeburg.de
}

\begin{abstract}
In this paper we discuss the usage of Accepting Networks of Evolutionary Processors (ANEPs for short) as deciding devices. In this context we define a new halting condition for this model, which seems more coherent with the rest of the theory than the previous such definition, and show that all the computability results reported so far remain valid in the new framework. Moreover, we give a direct and efficient simulation of an arbitrary ANEP by a complete ANEP, thus, showing that the efficiency of deciding a language by ANEPs is not influenced by the network's topology. Finally, we obtain a surprising characterization of $\mathbf{P}^{\mathbf{N P}[\log ]}$ as the class of languages that can be decided in polynomial time by ANEPs.
\end{abstract}

\section{Introduction}

The accepting networks of evolutionary processors (ANEPs, for short) are a bio-inspired computational mode, introduced in [12], and having its roots in [6, 3]. An ANEP consists in a graph having in each node a processor, which is able to perform very simple operations, namely point mutations in a DNA sequence (insertion, deletion or substitution of nucleotides); these processors are called evolutionary processor. Furthermore, each node contains data, which are organized in the form of multisets of words, each word appearing in an arbitrarily large number of copies, and all copies are processed in parallel such that all the possible events that can take place do actually take place. Following the biological motivation, each node may be viewed as a cell containing genetic information encoded in DNA sequences, which may evolve by local evolutionary events, i.e., point mutations; moreover, each node is specialized just for one of these evolutionary operations.

The computation of an ANEP is conducted as follows. Initially, only one special node, the input node, contains a certain word, the input word. Further, the words are processed in alternative evolutionary and communication steps. In an evolutionary step, the words found in each node are rewritten according to the rules of that node. In a communication step, the words of a node are communicated to the other nodes, as permitted by some filtering condition associated with both the sending and the receiving node. The classical definition assumes that a computation halts and accepts, when a word enters a special node of the network, the output

\footnotetext{
${ }^{(A)}$ Also at: Faculty of Mathematics and Computer Science, University of Bucharest, Str. Academiei 14, RO010014 Bucharest, Romania (flmanea@fmi .unibuc.ro). The work of Florin Manea is supported by the Alexander von Humboldt Foundation.
} 
node, or halts and rejects, when the words contained in each node do not change consecutive evolutionary or communication steps.

In this paper we propose a new halting condition for such a computation. Namely, a computation halts (and is called finite) as soon as at least one word enters the output node. The input word is accepted if the computation of the ANEP on this word is finite, and at least one word that is found in the output node at the end of the computation contains a special symbol; otherwise, the computation is either infinite, or, when it is finite, rejecting. The motivations behind this new halting condition are discussed in Section 3 .

Results on ANEPs, seen as formal languages accepting devices, were surveyed recently in [11]. In this paper we see how these results change when the new halting condition is used. While the computational power of the ANEPs remains the same, the time complexity results are not preserved. To this end, we obtain a surprising characterization of $\mathbf{P}^{\mathbf{N P}[\log ]}$ as the class of languages that can be decided in polynomial time by ANEPs.

We also show that an arbitrary ANEP can be simulated efficiently by an ANEP with complete underlying graph. This answers a question from [2] and shows that one cannot expect to decide a language faster, using ANEPs with special topology, than when complete ANEPs are used.

For the full proofs of the results presented in this paper see [9].

\section{Basic definitions}

We start by summarizing the notions used throughout the paper; for all unexplained notions the reader is referred to [14]. An alphabet is a finite and nonempty set of symbols. The cardinality of a finite set $A$ is written card $(A)$. Any sequence of symbols from an alphabet $V$ is called word (word) over $V$. The set of all words over $V$ is denoted by $V^{*}$ and the empty word is denoted by $\lambda$. The length of a word $x$ is denoted by $|x|$ while $\operatorname{alph}(x)$ denotes the minimal alphabet $W$ such that $x \in W^{*}$. For a word $x \in W^{*}, x^{r}$ denotes the reversal of the word.

In the following, we introduce a series of rewriting operations, called evolutionary operations as they may be viewed as linguistic formulations of local gene mutations. We say that a rule $a \rightarrow b$, with $a, b \in V \cup\{\lambda\}$ is a substitution rule if both $a$ and $b$ are not $\lambda$; it is a deletion rule if $a \neq \lambda$ and $b=\lambda$; it is an insertion rule if $a=\lambda$ and $b \neq \lambda$. The set of all substitution, deletion, and insertion rules over an alphabet $V$ are denoted by $S u b_{V}, D e l_{V}$, and $I_{n} s_{V}$, respectively.

Given a rule $\sigma$ as above and a word $w \in V^{*}$, we define the following actions of $\sigma$ on $w$ :

If $\sigma \equiv a \rightarrow b \in S u b_{V}$, then $\sigma^{*}(w)=\left\{\begin{array}{l}\left\{u b v \mid \exists u, v \in V^{*}(w=u a v)\right\}, \\ \{w \mid w \text { contains no } a\}\end{array}\right.$

If $\sigma \equiv a \rightarrow \lambda \in \operatorname{Del}_{V}$, then: $\sigma^{*}(w)=\left\{\begin{array}{l}\left\{u v \mid \exists u, v \in V^{*}(w=u a v)\right\} \text {, } \\ \{w \mid w \text { contains no } a\},\end{array}\right.$

$\sigma^{r}(w)=\left\{\begin{array}{l}\{u\}, w=u a, \\ \{w\}, \text { otherwise, }\end{array} \sigma^{l}(w)=\left\{\begin{array}{l}\{v\}, w=a v, \\ \{w\}, \text { otherwise, }\end{array}\right.\right.$

If $\sigma \equiv \lambda \rightarrow a \in \operatorname{Ins}_{V}$, then

$\sigma^{*}(w)=\left\{u a v \mid \exists u, v \in V^{*}(w=u v)\right\}, \sigma^{r}(w)=\{w a\}, \sigma^{l}(w)=\{a w\}$.

We say that $\alpha \in\{*, l, r\}$ expresses the way of applying a deletion or insertion rule to a word, namely at any position $(\alpha=*)$, in the left $(\alpha=l)$, or in the right $(\alpha=r)$ end of the word, respectively. For every rule $\sigma$, action $\alpha \in\{*, l, r\}$, and $L \subseteq V^{*}$, we define the $\alpha$-action of $\sigma$ 
on $L$ by $\sigma^{\alpha}(L)=\bigcup_{w \in L} \sigma^{\alpha}(w)$. Given a finite set of rules $M$, we define the $\alpha$-action of $M$ on the word $w$ and the language $L$ by $M^{\alpha}(w)=\bigcup_{\sigma \in M} \sigma^{\alpha}(w)$ and $M^{\alpha}(L)=\bigcup_{w \in L} M^{\alpha}(w)$, respectively.

For two disjoint subsets $P$ and $F$ of an alphabet $V$ and a word $w$ over $V$, we define the predicates:

$$
\begin{aligned}
& \varphi^{(s)}(w ; P, F) \equiv \quad P \subseteq \operatorname{alph}(w) \quad \wedge \quad F \cap \operatorname{alph}(w)=\emptyset \\
& \varphi^{(w)}(w ; P, F) \equiv \operatorname{alph}(w) \cap P \neq \emptyset \quad \wedge \quad F \cap \operatorname{alph}(w)=\emptyset .
\end{aligned}
$$

The construction of these predicates is based on random-context conditions defined by the two sets $P$ (permitting contexts/symbols) and $F$ (forbidding contexts/symbols). Informally, the first condition requires that all permitting symbols are present in $w$ and no forbidding symbol is present in $w$, while the second one is a weaker variant of the first, requiring that at least on permitting symbol appears in $w$ and no forbidding symbol is present in $w$.

For every language $L \subseteq V^{*}$ and $\beta \in\{(s),(w)\}$, we define:

$$
\varphi^{\beta}(L, P, F)=\left\{w \in L \mid \varphi^{\beta}(w ; P, F)\right\} .
$$

An evolutionary processor over $V$ is a tuple $(M, P I, F I, P O, F O)$, where:

- $M$ is a set of substitution, deletion or insertion rules over the alphabet $V$, the set of rules of the processor. Formally $\left(M \subseteq S u b_{V}\right)$ or $\left(M \subseteq D e l_{V}\right)$ or $\left(M \subseteq I n s_{V}\right)$. A processor is "specialized" in one type of operations only.

$-P I, F I \subseteq V$ are the input permitting, respectively forbidding, contexts of the processor, while $P O, F O \subseteq V$ are the output permitting, respectively forbidding, contexts of the processor. Informally, the permitting input (output) contexts are the set of symbols that should be present in a word, when it enters (respectively, leaves) the processor, while the forbidding contexts are the set of symbols that should not be present in a word in order to enter (respectively, leave) the processor.

- We denote the set of evolutionary processors over $V$ by $E P_{V}$.

Obviously, the evolutionary processor described here is a mathematical concept, inspired from the Darwinian evolution; we are not concerned here with a possible biological implementation of these processors, though a matter of great importance.

Next we define the central notion of our paper, namely Accepting Networks of Evolutionary Processors (ANEPs for short). Our definition is slightly different from the one that was used in literature so far (see [11] for a survey) by the usage of a special accepting symbol $\mu$.

The main reason for giving a different definition is that we are interested in analyzing such networks as deciding devices (thus, devices that halt on every input), not only as accepting devices. To this end, we feel that the halting conditions used in the previous definitions of ANEPs were somehow artificial, and quite unrelated to all the other concepts defined for these devices. We will motivate more our definition later, and the rest of the paper will be focused on analyzing the computational properties of the newly defined variant of ANEPs.

An accepting hybrid network of evolutionary processors (ANEP for short) is a 9-tuple $\Gamma=$ $\left(V, U, \mu, G, \mathcal{N}, \alpha, \beta, x_{I}, x_{O}\right)$, where:

- $V$ and $U$ are the input and network alphabets, respectively, $V \subseteq U$; the symbol $\mu \in U \backslash V$ is a distinguished symbol, called accepting symbol.

$-G=\left(X_{G}, E_{G}\right)$ is a directed graph, with the set of nodes $X_{G}$ and the set of edges $E_{G} \subseteq$ $X_{G} \times X_{G}$. The graph $G$ is called the underlying graph of the network, and card $\left(X_{G}\right)$ is the size of $\Gamma$.

$-\mathcal{N}: X_{G} \longrightarrow E P_{U}$ is a mapping which associates with each node $x \in X_{G}$ the evolutionary 
processor $\mathcal{N}(x)=\left(M_{x}, P I_{x}, F I_{x}, P O_{x}, F O_{x}\right)$.

$-\alpha: X_{G} \longrightarrow\{*, l, r\} ; \alpha(x)$ gives the action mode of the rules of node $x$ on the words existing in that node.

$-\beta: X_{G} \longrightarrow\{(s),(w)\}$ defines the type of the input/output filters of a node. More precisely, for every node, $x \in X_{G}$, the following filters are defined:

$$
\begin{gathered}
\text { input filter: } \rho_{x}(\cdot)=\varphi^{\beta(x)}\left(\cdot ; P I_{x}, F I_{x}\right), \\
\text { output filter: } \tau_{x}(\cdot)=\varphi^{\beta(x)}\left(\cdot ; P O_{x}, F O_{x}\right) \text {. }
\end{gathered}
$$

That is, $\rho_{x}(w)$ (respectively, $\tau_{x}(w)$ ) indicates whether or not the word $w$ can pass the input (respectively, output) filter of $x$.

$-x_{I}$ and $x_{O} \in X_{G}$ are the input node, and, respectively, the output node of $\Gamma$.

An ANEP is said to be complete if the underlying graph $G$ has the edges $E_{G}=\{(x, y) \mid x \neq y$ and $\left.x, y \in X_{G}\right\}$.

A configuration of an ANEP $\Gamma$ is a mapping $C: X_{G} \longrightarrow 2^{V^{*}}$, associating a set of words with every node of the graph. A configuration may be understood as the sets of words which are present in any node at a given moment; it can change either by an evolutionary step or by a communication step.

When changing by an evolutionary step each component $C(x)$ of the configuration $C$ is changed in accordance to the set of evolutionary rules $M_{x}$, of node $x$, and $\alpha(x)$, the way these rules should be applied. Formally, the configuration $C^{\prime}$ is obtained in one evolutionary step from the configuration $C$, written as $C \Longrightarrow C^{\prime}$, if and only if $C^{\prime}(x)=M_{x}^{\alpha(x)}(C(x))$, for all $x \in X_{G}$.

When changing by a communication step, each node-processor $x \in X_{G}$ sends one copy of each word it contains, and is able to pass its output filter, to all the node-processors connected to $x$, and receives all the words sent by all the other node processor connected with $x$, provided that they can pass its input filter. Formally, the configuration $C^{\prime}$ is obtained in one communication step from configuration $C$, written as $C \vdash C^{\prime}$, if and only if $C^{\prime}(x)=\left(C(x)-\tau_{x}(C(x))\right) \cup$ $\bigcup_{(y, x) \in E_{G}} \rho_{x}\left(\tau_{y}(C(y))\right)$, for all $x \in X_{G}$. Note that the words which leave a node are eliminated from that node; if such a word cannot pass the input filter of any node, it is lost.

The computation of $\Gamma$ on the input word $w \in V^{*}$ is a (potential infinite) sequence of configurations $C_{0}^{(w)}, C_{1}^{(w)}, C_{2}^{(w)}, \ldots$ The initial configuration $C_{0}^{(w)}$ is defined by $C_{0}^{(w)}\left(x_{I}\right)=\{w\}$ and $C_{0}^{(w)}(x)=\emptyset$ for all $x \in X_{G}, x \neq x_{I}$. Further, $C_{2 i}^{(w)} \Longrightarrow C_{2 i+1}^{(w)}$ and $C_{2 i+1}^{(w)} \vdash C_{2 i+2}^{(w)}$, for all $i \geq 0$.

The acceptance symbol $\mu$ is important when defining the accepting computations of an ANEP. A computation as above halts when it reaches a configuration $C_{t}^{w}$ in which the output node $x_{O}$ contains a word; we say that $\Gamma$ halts on the input word $w$. We distinguish two situations: i. There exists $u \in C_{t}^{w}\left(x_{O}\right)$, such that $u$ contains the symbol $\mu$. In this case, the computation is an accepting computation, and $\Gamma$ accepts $w$.

ii. Any word $u \in C_{t}^{w}\left(x_{O}\right)$ does not contain the symbol $\mu$. In this case, the computation is a rejecting computation, and $\Gamma$ rejects $w$.

The language accepted by $\Gamma$ is $L_{a}(\Gamma)=\left\{w \in V^{*} \mid\right.$ the computation of $\Gamma$ on $w$ is an accepting one . We say that an ANEP $\Gamma$ decides the language $L \subseteq V^{*}$, and write $L(\Gamma)=L$ if and only if $L_{a}(\Gamma)=L$ and $\Gamma$ halts on all input words.

Let $\Gamma$ be an ANEP deciding the language $L$.

The time complexity of the finite computation $C_{0}^{w}, C_{1}^{w}, C_{2}^{w}, \ldots C_{m}^{w}$ of $\Gamma$ on $w \in L$ is denoted by $\operatorname{Time}_{\Gamma}(w)$ and equals $m$. The time complexity of $\Gamma$ is the function $\operatorname{Time}_{\Gamma}(n)=$ 
$\max \left\{\operatorname{Time}_{\Gamma}(x)|| x \mid=n\right\}$. We say that $\Gamma$ works in polynomial time, if there exists a polynomial function $f$ such that $f(n) \geq \operatorname{Time}_{\Gamma}(n)$.

For a function $f: \mathbf{N} \longrightarrow \mathbf{N}$ we define Time ANEP $(f(n))=\{L \mid$ there exists the ANEP $\Gamma$, such that $L(\Gamma)=L$ Time $_{\Gamma}(n) \leq f(n)$, for all $\left.n \in I N\right\}$. Moreover, we write PTime $A N E P=$ $\bigcup_{k \geq 0}$ Time $_{A N E P}\left(n^{k}\right)$.

One can define in a similar manner space and length complexity classes ([11]).

\section{The new halting condition and computability results}

The single difference between the initial definition of ANEPs ([12]) and ours is the presence and usage of the symbol $\mu$. The way a computation of an ANEP is conducted remains basically the same, but the halting conditions (both in the case of acceptance and rejection) are essentially different.

But let us first recall the definition of a halting ANEP-computation from the literature (see the seminal work [12], the survey [11], and the references therein). A computation halts and accepts if there exists a configuration in which the set of words existing in the output node is non-empty. A computation halts and rejects if there exist two identical configurations obtained either in consecutive evolutionary steps or in consecutive communication steps. The language accepted by the ANEP $\Gamma$ is $L_{a}(\Gamma)=\left\{w \in V^{*} \mid\right.$ the computation of $\Gamma$ on $w$ accepts $\}$. Also, it was said that an ANEP $\Gamma$ decides the language $L \subseteq V^{*}$ iff $L_{a}(\Gamma)=L$ and $L$ halts on every input.

The reasons that made us switch to the new definition are related mainly to the rejecting computations.

First, the rejecting condition did not seem coherent with the other conditions verified in a ANEP. The filters verify the existence or absence of several symbols in the communicated words; the application of a rule by a processor consists (in the more complicated cases of substitution and deletion rules) in looking for the occurrences of a symbol in the words of that node (if it contains any), and replacing an arbitrary such occurrence with a symbol or with $\lambda$. The rejecting condition assumed a very different process: one checked whether the configurations of all the nodes, in two consecutive steps of the same kind, were equal.

Also, the processes executed by an ANEP are localized: filters are associated with nodes, rules are associated with nodes, and the accepting condition concerned only one node, the output node. In the case of a rejecting computation the definition took us to a global level: we looked at all the words present at a given moment in the network. The condition seemed an artificial formalization of the case when the network enters in an infinite loop, and the computation should halt. However, only infinite loops in which the configurations are repeated in consecutive steps were detected. Although avoiding infinite loops seems to us a good-practice in programming (regardless of the computational model), we do not see a reason for it to be ruled out from the definition.

Nevertheless, verifying the equality between two configurations required to memorize, at any moment, all the words from the last two configurations. Thus, an additional memory-device was needed, and this was not (explicitly) part of an ANEP. This affected the self-containment of the definition.

The new halting conditions seem to overcome these problems. The computation halts as soon as a word enters in a special node. Although it seems to be also a condition of a different 
nature from the ones that are checked in an ANEP, it is natural to think that before each processing step a processor checks whether it contains some words, and then it looks for the places where the rules can be applied. Further, the decision of a computation is taken according to a test in which we check for the existence of a symbol in the words of a node; this seems coherent with the rest of the definition of a ANEP. Moreover, we do not use any auxiliary devices (as it was the memory we needed in the former case).

It only remains to be settled in which measure the results already reported for ANEPs ([11] $)$ still hold, with respect to the new definition.

Accepting a language. All the ANEP constructions proposed in the literature (for instance, in [10, 7, 1]), where one was interested only in accepting a language by complete ANEPs, can be still be used. However, we must modify such an ANEP in order to work properly in the new setting: the former output node becomes an insertion node where the symbol $\mu$ is inserted in the words that were accepted inside, and then we add a new output node, in which all the words containing $\mu$ are allowed to enter. Thus, one can construct, for a recursively enumerable language, an ANEP accepting it, w.r.t. the new definition.

Deciding a language. In [10] one shows that the class of languages decided by an ANEP, with respect to the classical halting condition, is the class of recursive languages. The proof was based on simulating, in parallel, all the possible computations of a nondeterministic Turing machine; the words communicated in the network were encodings of the Turing machine configurations. As we have already mentioned, these proofs can be used, as long as we are not interested in deciding the language, but only in accepting it. However, any recursive language can be decided by a deterministic Turing machine that for each input either enters a single final state and accepts, or enters a single blocking state and rejects. The ANEP simulating a Turing machine, presented in [10], can be easily modified to decide a recursive language $L$ : we simulate the deterministic Turing machine deciding $L$ and allow in the former output node all the words that contain the final state or the blocking state; if a word containing the final state enters the output node, then the network accepts, otherwise, it rejects. In conclusion, the languages decided by ANEPs, w.r.t. the new definition, are the recursive languages.

Computational Complexity. The results regarding polynomial space complexity or polynomial deterministic time complexity reported [10] can be also proved by simulating deterministic Turing machines by ANEPs and vice versa. Therefore, they remain valid when the new acceptance/rejection conditions are used. However, the results that were based on time-efficient simulations of nondeterministic machines are not preserved, as we will see in Section 5 .

\section{Complete ANEPs}

It is worth mentioning that most of the computability and computational complexity results reported so far in literature deal with complete ANEPs or with ANEPs with a restricted topology (see [11] and the references therein). More precisely, there are many results stating that particular types of networks are accepting all the recursively enumerable languages or perform a series of tasks efficiently (e.g. solving NP-complete problems, or simulating efficiently different types of universal devices).

A natural question arises: in which measure is the topology of the networks important, with respect to the computational power or the efficiency of the computations? Such a result 
would be interesting if we consider that sometimes it is easier to construct an ANEP with a particular topology, solving a given problem efficiently, than to construct a complete one (thus, it is simpler to solve a problem by a non-uniform approach than by an uniform one). For instance, in [2] it was left as an open problem to see if the reported results still hold for complete ANEPs.

A first answer is immediate, but unsatisfactory. Complete ANEPs can simulate (with respect to the former halting conditions) nondeterministic Turing machines, and nondeterministic Turing machines can simulate ANEPs of any kind (see [10]). So one can construct a complete ANEP simulating an arbitrary ANEP via the simulation by Turing machines. However, such a simulation is not time-efficient since the Turing machine simulates a computation of $t$ steps of the ANEP on an input word of length $n$ in time $\mathcal{O}\left(\max \left(t^{2}, t n\right)\right)$; this approach is also complicated due to the the construction of the intermediate Turing machine. Such an approach leads to complete ANEPs that solve quite inefficiently a given problem (see, for instance, Example 5.5.).

In the following we propose a new answer to the above question: we can accept (respectively, decide) with a complete ANEP any language accepted (respectively, decided) by an ANEP with an arbitrary underlying graph, within the same computing time. The new halting conditions play an important role. As we have already explained, they are not relevant in the case when we are interested only in accepting languages: if we use these conditions, we still obtain that given an arbitrary ANEP one can construct a complete ANEP accepting, as efficiently as the arbitrary ANEP, the same language. They come into play in the case of deciding languages. Basically, the proof of our result consists in simulating an ANEP by a complete ANEP. Two consecutive steps of the initial ANEP are simulated in exactly 54 consecutive steps of the complete ANEP. In the classical setting, the initial ANEP rejected when the configurations obtained in two consecutive steps of the same kind were identical; but these configurations do not occur in the complete ANEP consecutively so the new network would not reject, but enter in an infinite cycle. Thus, such a simulation would not preserve the halting property of a computation. However, when the new conditions are used the halting property is preserved canonically.

The proof of the announced results is based on the following two Lemmas.

Lemma 4.1 Given an $A N E P \Gamma=(V, U, \mu, G, \mathcal{N}, \alpha, \beta$, In, Out $)$, one can construct an ANEP $\Gamma^{\prime}=\left(V, U, \mu, G^{\prime}, \mathcal{N}^{\prime}, \alpha^{\prime}, \beta^{\prime}, I n^{\prime}\right.$, Out $\left.{ }^{\prime}\right)$ such that $\Gamma^{\prime}$ accepts (decides) the same language as $\Gamma$ accepts (respectively, decides), each node of $\Gamma^{\prime}$ has at most one rule and $I n^{\prime}$ has no rules. Moreover, two consecutive steps of $\Gamma$ (an evolutionary and a communication step) are simulated in exactly 6 consecutive steps ( 3 evolutionary and 3 communication steps) of $\Gamma^{\prime}$.

Proof. We will show how we can construct for each node $x$, of the network $\Gamma$, a subnetwork $s(x)$, contained in $\Gamma^{\prime}$, that simulates the computation of the processor $\mathcal{N}(x)$. We denote by $\operatorname{set}(s(x))$ the nodes of the subnetwork $s(x)$, and distinguish two nodes of this subnetwork, namely $i(s(x))$ and $o(s(x))$, that make possible the communication with the subnetworks constructed for the other nodes (these two nodes can be seen as the input node, and, respectively, the output node of the network, while all the other nodes can be seen as internal nodes). We also denote by edge $(s(x))$ the set of edges of the subnetwork (that is, the edges connecting the nodes of the subnetwork).

Let us assume that the node $x$ verifies $\mathcal{N}(x)=(M, P I, F I, P O, F O), M=\left\{r_{1}, \ldots, r_{n}\right\}$, and $\alpha(x)=s$. Then we have: 
- $\operatorname{set}(s(x))=\left\{x_{0}, x_{0}^{\prime}, x_{1}, x_{2}\right\} \cup\left\{x_{a} \mid a \in P O\right\} \cup\left\{x_{1}^{r}, \ldots, x_{n}^{r}\right\}, i(s(x))=x_{0}, o(s(x))=x_{2}$.

- edge $(s(x))=\left\{\left(x_{a}, x_{0}^{\prime}\right),\left(x_{i}^{r}, x_{a}\right) \mid a \in P O, i \in\{1, \ldots, n\}\right\} \cup\left\{\left(x_{0}, x_{i}^{r}\right),\left(x_{i}^{r}, x_{1}\right),\left(x_{i}^{r}, x_{2}\right)\right.$, $\left.\left(x_{1}, x_{0}^{\prime}\right),\left(x_{0}^{\prime}, x_{i}^{r}\right) \mid i \in\{1, \ldots, n\}\right\}$.

- $\mathcal{N}^{\prime}\left(x_{0}\right)=(\emptyset, P I, F I, \emptyset, \emptyset), \alpha^{\prime}\left(x_{1}\right)=s, \beta^{\prime}\left(x_{1}\right)=*$.

- $\mathcal{N}^{\prime}\left(x_{1}\right)=(\emptyset, F O, \emptyset, U, \emptyset), \alpha^{\prime}\left(x_{1}\right)=w, \beta^{\prime}\left(x_{1}\right)=*$.

- For $a \in P O$ we have: $\mathcal{N}^{\prime}\left(x_{a}\right)=(\emptyset, W,\{a\}, U, \emptyset), \alpha^{\prime}\left(x_{a}\right)=w, \beta^{\prime}\left(x_{a}\right)=*$.

- For $i \in\{1, \ldots, n\}$ we have: $\mathcal{N}^{\prime}\left(x_{i}^{r}\right)=\left(\left\{r_{i}\right\}, U, \emptyset, U, \emptyset\right), \alpha^{\prime}\left(x_{i}^{r}\right)=w, \beta^{\prime}\left(x_{i}^{r}\right)=*$.

- $\mathcal{N}^{\prime}\left(x_{0}^{\prime}\right)=(\emptyset, U, \emptyset, U, \emptyset), \alpha^{\prime}\left(x_{0}^{\prime}\right)=w, \beta^{\prime}\left(x_{0}^{\prime}\right)=*$.

- $\mathcal{N}^{\prime}\left(x_{2}\right)=(\emptyset, P O, F O, \emptyset, \emptyset), \alpha^{\prime}\left(x_{2}\right)=s, \beta^{\prime}\left(x_{2}\right)=*$.

To see that the subnetwork $s(x)$ defined above simulates the behavior of the node $x$, let us assume that $w$ is a word that was sent towards the node $x$ in a communication step of $\Gamma$, and the same word was also sent towards $x_{0}$ in $\Gamma^{\prime}$. Of course $w$ enters $x$ if and only if it enters $x_{0}$. In $x$, a rule $r_{i}$ is applied to $w$; this is simulated in $s(x)$ in the following steps: $w$ goes from $x_{0}$ to $x_{i}^{r}$, where the rule $r_{i}$ is applied to it. Back in $\Gamma$, the word can exit the node $x$, if it verifies the filters $P O$ and $F O$, or remain in the node and be further processed. In $\Gamma^{\prime}$ the word can go to $x_{2}$, if it verifies the filters $P O$ and $F O$, and leave the subnetwork in the next communication step; if it does not verify these filters, it goes to $x_{1}$ (if it has a forbidden symbol in it) or into a node $x_{a}$ (if it does not contain the symbol $a \in P O$ ), and from these nodes is sent to $x_{0}^{\prime}$, and the it is resent to the nodes $x_{j}^{r}$, for $j \in\{1, \ldots, n\}$, to be further processed. It is clear that the subnetwork $s(x)$ behaves exactly like the node $x$. Moreover, one processing step and one communication step of $\Gamma$ are simulated in $\Gamma^{\prime}$ by 3 processing and 3 communication steps.

The case of the nodes with weak filters is a easier. Let us assume that the node $x$ verifies $\mathcal{N}(x)=(M, P I, F I, P O, F O), M=\left\{r_{1}, \ldots, r_{n}\right\}$, and $\alpha(x)=w$. Then we have:

- $\operatorname{set}(s(x))=\left\{x_{0}, x_{1}, x_{1}^{\prime}, x_{2}\right\} \cup\left\{x_{1}^{r}, \ldots, x_{n}^{r}\right\}, i(s(x))=x_{0}, o(s(x))=x_{2}$.

- edge $(s(x))=\left\{\left(x_{0}, x_{i}^{r}\right),\left(x_{i}^{r}, x_{1}\right),\left(x_{i}^{r}, x_{2}\right),\left(x_{0}^{\prime}, x_{i}^{r}\right),\left(x_{i}^{r}, x_{1}^{\prime}\right), \mid i \in\{1, \ldots, n\}\right\} \cup$ $\cup\left\{\left(x_{1}, x_{0}^{\prime}\right),\left(x_{1}^{\prime}, x_{0}^{\prime}\right)\right\}$.

- $\mathcal{N}^{\prime}\left(x_{0}\right)=(\emptyset, P I, F I, U, \emptyset), \alpha^{\prime}\left(x_{0}\right)=w, \beta^{\prime}\left(x_{0}\right)=*$.

- $\mathcal{N}^{\prime}\left(x_{1}\right)=(\emptyset, F O, \emptyset, U, \emptyset), \alpha^{\prime}\left(x_{1}\right)=w, \beta^{\prime}\left(x_{1}\right)=*$.

- $\mathcal{N}^{\prime}\left(x_{1}^{\prime}\right)=(\emptyset, W, P O, U, \emptyset), \alpha^{\prime}\left(x_{a}\right)=w, \beta^{\prime}\left(x_{a}\right)=*$.

- For $i \in\{1, \ldots, n\}$ we have: $\mathcal{N}^{\prime}\left(x_{i}^{r}\right)=\left(\left\{r_{i}\right\}, U, \emptyset, U, \emptyset\right), \alpha^{\prime}\left(x_{i}^{r}\right)=w, \beta^{\prime}\left(x_{i}^{r}\right)=*$.

- $\mathcal{N}^{\prime}\left(x_{0}^{\prime}\right)=(\emptyset, U, \emptyset, U, \emptyset), \alpha^{\prime}\left(x_{0}^{\prime}\right)=w, \beta^{\prime}\left(x_{0}^{\prime}\right)=*$.

- $\mathcal{N}^{\prime}\left(x_{2}\right)=(\emptyset, P O, F O, U, \emptyset), \alpha^{\prime}\left(x_{2}\right)=w, \beta^{\prime}\left(x_{2}\right)=*$.

The simulation of the computation of node $x$ by the subnetwork $s(x)$ goes pretty much like the above. The only difference is that in the case of weak filters, a word cannot exit $x$ in the case when it contains a forbidden symbol (in the simulation, the word goes to $x_{1}$ ) or in the case it does not contain any of the permitting symbols (in the simulation, the word goes to $x_{1}^{\prime}$ ). As in the former case, one processing step and one communication step of $\Gamma$ are simulated in $\Gamma^{\prime}$ by 3 processing and 3 communication steps.

If a node $x$ has no rules, our intuition is that it should be kept in the exact same form in the new network. However, in order to be sure that the ANEP we construct simulates correctly the 
given one, we must be sure that each step of the initial network is simulated in the same number of steps by the new one. For this, we must be sure that an evolutionary step of the initial network, in which no rule are applied, and the consequent communication step are simulated in 3 processing (in which no rule will be applied) and 3 communication steps of the new network. Therefore, we set, in the case of $\alpha(x)=s$ :

- $\operatorname{set}(s(x))=\left\{x_{0}, x_{1}, x_{2}\right\}, i(s(x))=x_{0}, o(s(x))=x_{2}$.

- $\operatorname{edge}(s(x))=\left\{\left(x_{0}, x_{1}\right),\left(x_{1}, x_{2}\right)\right\}$.

- $\mathcal{N}^{\prime}\left(x_{0}\right)=(\emptyset, P I, F I, U, \emptyset), \alpha^{\prime}\left(x_{0}\right)=s, \beta^{\prime}\left(x_{0}\right)=*$.

- $\mathcal{N}^{\prime}\left(x_{1}\right)=(\emptyset, P O, F O, U, \emptyset), \alpha^{\prime}\left(x_{1}\right)=s, \beta^{\prime}\left(x_{1}\right)=*$.

- $\mathcal{N}^{\prime}\left(x_{2}\right)=(\emptyset, U, \emptyset, U, \emptyset), \alpha^{\prime}\left(x_{2}\right)=w, \beta^{\prime}\left(x_{2}\right)=*$.

The case of weak filters can be treated in the same manner: we just have to change the permitting output filters of $x_{0}$ and $x_{1}$, and make them equal to $U$, and set $\alpha\left(x_{0}\right)=\alpha\left(x_{1}\right)=s$.

To finish the construction of the network $\Gamma^{\prime}$ we set:

- $G^{\prime}=\left(X_{G^{\prime}}, E_{G^{\prime}}\right)$, where $X_{G^{\prime}}=\bigcup_{x \in X_{G}} \operatorname{set}(s(x))$ and $E_{G^{\prime}}=\{(o(s(x)), i(s(y))) \mid(x, y) \in$ $\left.E_{G}\right\} \cup \cup_{x \in X_{G}} \operatorname{edge}(s(x))$

- The input node of the network, denoted $x_{I}^{\prime}$, is $i\left(s\left(x_{I}\right)\right)$. The output node of the network, denoted $x_{O}^{\prime}$, is $i\left(s\left(x_{O}\right)\right)$.

From the remarks made when we explained our simulations it is clear that the network $\Gamma^{\prime}$ accepts (decides) exactly the same language as $\Gamma$.

Lemma 4.2 Given an ANEP $\Gamma=(V, U, \mu, G, \mathcal{N}, \alpha, \beta$, In, Out $)$, such that all the processors $\Gamma$ have at most one rule and In has no rules, one can construct a complete ANEP $\Gamma^{\prime}=(V$, $U^{\prime}, \mu, \nu, G^{\prime}, \mathcal{N}^{\prime}, \alpha^{\prime}, \beta^{\prime}, I n^{\prime}$, Out') such that $\Gamma^{\prime}$ accepts (decides) the same language as $\Gamma$ accepts (respectively, decides). Moreover, two consecutive steps of $\Gamma$ (an evolutionary and a communication step) are simulated in exactly 18 consecutive steps (9 evolutionary and 9 communication steps) of $\Gamma^{\prime}$.

Proof. Let $U_{1}=\left\{\#_{x}, \#_{x}^{\prime}, \#_{x}^{\prime \prime}, \#_{x}^{b}, \#_{x, y} \mid b \in U, x, y \in X_{G},(x, y) \in E_{G}\right\}$.

The complete network $\Gamma^{\prime}$ simulates the computation of $\Gamma$ using the following strategy. We try to construct for each node $x$, of $\Gamma$, a subnetwork $s(x)$, of $\Gamma^{\prime}$, that simulates the computation of the processor $\mathcal{N}(x)$; we denote by $\operatorname{set}(s(x))$ the nodes of the subnetwork $s(x)$. The underlying graph of $\Gamma^{\prime}$ is complete and has the nodes $\bigcup_{x \in X_{G}} \operatorname{set}(x)$. All the words processed in the new network have a special symbol from $U_{1}$. The symbols of $U_{1}$ that encode one processor of the initial network indicate the nodes whose actions must be simulated at that point, thus which of the subnetworks should act on the word. The symbols that encode two nodes indicate a possible way to communicate the word containing it between the subnetworks of $\Gamma^{\prime}$. The symbol $\#_{I n}$ is inserted in the input word at the beginning of the computation, so we should start by simulating the input node of $\Gamma$. Further, the way the computation is conducted, described below, and the way symbols of $U_{1}$ are inserted, deleted or modified, in the processed words enable us to simulate, in parallel, all the possible derivations of the input word in $\Gamma$, and ensure that the subnetworks act independently. 
The alphabet of $\Gamma^{\prime}$ is defined as $U^{\prime}=U \cup\left\{b^{\prime}, b^{\prime \prime} \mid b \in U\right\} \cup\left\{\#^{(i)} \mid 1 \leq i \leq 8\right\} \cup U_{1}$. In the following, we define the rest of the network.

We will split our discussion in many cases, according to the type of the node (with no rules, insertion, substitution, deletion), the way the operations are applied (left, right, arbitrary) and the type of the filters. We stress out from the beginning that one processing step and the subsequent communication step of the network $\Gamma$ will be simulated in exactly 9 processing steps and the corresponding communications steps in $\Gamma^{\prime}$; this is because in the most intricate case, namely the case of deletion nodes, our network actually needs this many steps to simulate correctly one step of $\Gamma$.

Assume that the node $x$ verifies $\mathcal{N}(x)=(\emptyset, P I, F I, P O, F O)$. Then we have:

- $\operatorname{set}(s(x))=\left\{x_{0}, x_{1}\right\}$,

- $\mathcal{N}\left(x_{0}\right)=\left(\left\{\#_{x} \rightarrow \#_{x}^{\prime}\right\}, P I, F I \cup\left(U_{1} \backslash\left\{\#_{x}\right\}\right),\left\{\#_{x}^{\prime}\right\}, \emptyset\right), \alpha^{\prime}\left(x_{0}\right)=\alpha(x)$.

- $\mathcal{N}\left(x_{1}\right)=\left(\left\{\#_{x}^{\prime} \rightarrow \#^{(1)}, \#^{(7)} \rightarrow \#_{y} \mid(x, y) \in E_{G}\right\} \cup\left\{\#^{(i)} \rightarrow \#^{(i+1)} \mid 1 \leq i \leq 6\right\}, U^{\prime}, \emptyset, P O, F O \cup\right.$ $\left.\left\{\#_{x}^{\prime}\right\} \cup\left\{\#^{(i)} \mid 1 \leq i \leq 7\right\}\right), \alpha^{\prime}\left(x_{1}\right)=\alpha(x)$.

In $s(x)$ we only change the symbol $\#_{x}$ into a new symbol $\#_{y}$, indicating that the word can now go towards the nodes of the subnetwork $s(y)$, and cannot enter the nodes of any other subnetwork. The only trick is that we must do this change in nine steps, instead of a single rewriting step. The rest of the word is left unchanged, as it was also the case in the node $x$ of the initial network, where the whole word stayed unchanged.

In the case of the input node $I n$ of $\Gamma$, the only difference is that we add a new node $I n_{0}^{\prime}$, which is an insertion node, where $\#_{I n}^{\prime}$ is inserted. The input node of the network is $I n_{0}^{\prime}$. This node does not allow any word to enter, and allows all the words to exit. The subnetwork associated with the output node Out has only the node Out $t_{0}$, which is the output node of the new network.

For the node $x$, with $\mathcal{N}(x)=(\{\lambda \rightarrow a\}, P I, F I, P O, F O), \alpha(x)=s$ and $\beta(x)=l$, we have:

- $\operatorname{set}(s(x))=\left\{x_{0}, x_{1}, x_{2}, x_{3}\right\}$.

- $\mathcal{N}\left(x_{0}\right)=\left(\left\{\lambda \rightarrow \#_{x}^{\prime}\right\}, P I, F I \cup\left(U_{1} \backslash\left\{\#_{x}\right\}\right), U^{\prime}, \emptyset\right), \alpha^{\prime}\left(x_{0}\right)=s, \beta^{\prime}\left(x_{0}\right)=r$.

- $\mathcal{N}\left(x_{1}\right)=\left(\left\{\#_{x} \rightarrow \lambda\right\},\left\{\#_{x}^{\prime}, \#_{x}\right\}, U_{1} \backslash\left\{\#_{x}^{\prime}, \#_{x}\right\}, U^{\prime}, \emptyset\right), \alpha^{\prime}\left(x_{1}\right)=s, \beta^{\prime}\left(x_{1}\right)=*$.

- $\mathcal{N}\left(x_{2}\right)=\left(\left\{\lambda \rightarrow a^{\prime}\right\},\left\{\#_{x}^{\prime}\right\}, U_{1} \backslash\left\{\#_{x}^{\prime}\right\},\left(P O \cup\left\{a^{\prime}\right\}\right) \backslash\{a\}, F O \cup\left\{b^{\prime} \mid b \in F O\right\}\right), \alpha^{\prime}\left(x_{2}\right)=s$, $\beta^{\prime}\left(x_{2}\right)=l$.

- $\mathcal{N}\left(x_{3}\right)=\left(\left\{a^{\prime} \rightarrow a\right\} \cup\left\{\#_{x}^{\prime} \rightarrow \#^{(1)}, \#^{(1)} \rightarrow \#^{(2)}, \#^{(2)} \rightarrow \#^{(3)}, \#^{(3)} \rightarrow \#^{(4)}, \#^{(4)} \rightarrow \#_{y} \mid(x, y) \in\right.\right.$ $\left.\left.E_{G}\right\},\left\{a^{\prime}\right\}, U_{1} \backslash\left\{\#_{x}^{\prime}\right\},\left\{\#_{y} \mid(x, y) \in E_{G}\right\},\left\{a^{\prime}, \#_{x}^{\prime}\right\}\right), \alpha^{\prime}\left(x_{3}\right)=w, \beta^{\prime}\left(x_{3}\right)=*$.

The subnetwork associated with an right insertion node, with strong filters, is very similar, the only different things being that $\beta^{\prime}\left(x_{0}\right)=l$ and $\beta^{\prime}\left(x_{2}\right)=r$. Also, the subnetwork associated with an arbitrary insertion node, with strong filters, is similar, this time the different things being that $\beta^{\prime}\left(x_{0}\right)=\beta^{\prime}\left(x_{2}\right)=*$.

In the following we explain how the subnetwork works in the case of left insertion nodes (as the other cases are treated analogously). Let us assume that $w$ is a word that was sent towards the node $x$ in a communication step of $\Gamma$, and the word $w_{1} \#_{x} w_{2}$, with $w_{1} w_{2}=w$, was communicated in the network $\Gamma^{\prime}$. If the word $w$ can pass the input filters of $x$ then $w_{1} \#_{x} w_{2}$ can also enter $x_{0}$ (and no other node); the reversal holds also. In the node $x$ we obtain from $w$ the 
word $a w$, which leaves the node if it verifies the output conditions; note that if the word does not leave the node after one processing step, then it will never leave, since any new insertion would not make it contain new symbols (because, as we have assumed, we have at most one rule per node). In the network $\Gamma^{\prime}$ the word is processed as follows. In $x_{0}$ it becomes $w_{1} \#_{x} w_{2} \#_{x}^{\prime}$ and is sent out. As we will see after the whole network is defined, it can only enter $x_{1}$ (because all the other nodes block the words containing both $\#_{x}$ and $\#_{x}^{\prime}$ ); here it becomes $w_{1} w_{2} \#_{x}^{\prime}=w \#_{x}^{\prime}$. Further, it is communicated in the network and can only enter $x_{2}$, where it becomes $a^{\prime} w \#_{x}^{\prime}$. It is not hard to see that this word can leave the node $x_{2}$ if and only if aw can leave the node $x$; as in the case of the initial network, if the word does not leave the node after one processing step, then it will never do so. Therefore, if the word verifies the output filters and leaves the node it can only go to node $x_{3}$ where we obtain, in 6 processing steps, all the words $a w \#_{y}$, for a node $y$ such that $(x, y) \in E_{G}$. Each word $a w \#_{y}$ leaves the node and can go to the nodes of the subnetworks associated with the node $y$. Clearly, $s(x)$ simulates correctly one processing step done by $x$, and the consequent communication step, in 9 processing and communication steps.

The case of insertion nodes with weak filters is very similar. We just have to redefine the filters of $x_{0}$ and $x_{2}$ to weak, and to set to $U^{\prime}$ the permitting output filter of $x_{0}$.

Now we move on to substitution nodes. In this case we only have two cases, according to the way filters are used, since substitutions are always applied arbitrary. For the node $x$, with $\mathcal{N}(x)=(\{a \rightarrow b\}, P I, F I, P O, F O), a \in F O, \alpha(x)=s$ and $\beta(x)=*$, we have:

- $\operatorname{set}(s(x))=\left\{x_{0}, x_{1}, x_{2}, x_{3}, x_{4}, x_{5}, x_{6}\right\}$.

- $\mathcal{N}\left(x_{0}\right)=\left(\left\{\#_{x} \rightarrow \#_{x}^{\prime}\right\}, P I, F I \cup\left(U_{1} \backslash\left\{\#_{x}\right\}\right), U^{\prime}, \emptyset\right), \alpha^{\prime}\left(x_{0}\right)=s, \beta^{\prime}\left(x_{0}\right)=*$.

- $\mathcal{N}\left(x_{1}\right)=\left(\left\{a \rightarrow b^{\prime}\right\},\{a\}, U_{1} \backslash\left\{\#_{x}^{\prime}\right\}, U^{\prime}, \emptyset\right), \alpha^{\prime}\left(x_{1}\right)=s, \beta^{\prime}\left(x_{1}\right)=*$.

- $\mathcal{N}\left(x_{2}\right)=\left(\left\{\#_{x}^{\prime} \rightarrow \#_{x}^{\prime \prime}\right\},\left\{\#_{x}^{\prime}\right\},\{a\} \cup\left(U_{1} \backslash\left\{\#_{x}^{\prime}\right\}\right), U^{\prime}, \emptyset\right), \alpha^{\prime}\left(x_{2}\right)=s, \beta^{\prime}\left(x_{3}\right)=*$.

- $\mathcal{N}\left(x_{3}\right)=\left(\left\{\#_{x}^{\prime} \rightarrow \#_{x}^{\circ}\right\},\left\{b^{\prime}\right\}, U_{1} \backslash\left\{\#_{x}^{\prime}\right\},\{a\}, F O \cup\left\{c^{\prime} \mid c \in F O\right\}\right), \alpha^{\prime}\left(x_{3}\right)=s, \beta^{\prime}\left(x_{3}\right)=*$.

- $\mathcal{N}\left(x_{4}\right)=\left(\left\{b^{\prime} \rightarrow b, \#_{x}^{\circ} \rightarrow \#^{(1)}, \#^{(1)} \rightarrow \#^{(2)}, \#^{(2)} \rightarrow \#^{(3)}, \#^{(3)} \rightarrow \#^{(4)}, \#^{(4)} \rightarrow \#_{x}^{\prime}\right\},\left\{b^{\prime}\right\}, U_{1} \backslash\right.$ $\left.\left\{\#_{x}^{\circ}\right\},\{a\}, F O \cup\left\{b^{\prime}, \#_{x}^{\circ}\right\}\right), \alpha^{\prime}\left(x_{4}\right)=w, \beta^{\prime}\left(x_{4}\right)=*$.

- $\mathcal{N}\left(x_{5}\right)=\left(\left\{b^{\prime} \rightarrow b, \#_{x}^{\prime} \rightarrow \#^{(2)}, \#_{x}^{\prime \prime} \rightarrow \#^{(1)}, \#^{(1)} \rightarrow \#^{(2)}, \#^{(2)} \rightarrow \#^{(3)}, \#^{(3)} \rightarrow \#^{(4)}, \#^{(4)} \rightarrow \#_{x, y} \mid\right.\right.$ $\left.b \in U,(x, y) \in E_{G}\right\},\left\{b^{\prime}, \#_{x}^{\prime \prime}\right\}, F O \cup\left\{c^{\prime} \mid c \in F O\right\} \cup\left(U_{1} \backslash\left\{\#_{x}^{\prime}, \#_{x}^{\prime \prime}\right\}\right), U^{\prime},\left\{\#^{(i)} \mid 1 \leq i \leq 4\right\} \cup$ $\left.\left\{\#_{x}^{\prime \prime}, b^{\prime}, \#_{x}^{\prime}\right\}\right), \alpha^{\prime}\left(x_{5}\right)=w, \beta^{\prime}\left(x_{5}\right)=*$.

- $\mathcal{N}\left(x_{6}\right)=\left(\left\{\#_{x, y} \rightarrow \#_{y} \mid Y \in X_{G}\right\}, P O, U_{1} \backslash\left\{\#_{x, y} \mid(x, y) \in E_{G}\right\}, U^{\prime},\left\{\#_{x, y} \mid(x, y) \in E_{G}\right\}\right)$, $\alpha^{\prime}\left(x_{6}\right)=s, \beta^{\prime}\left(x_{6}\right)=*$.

If $a \notin F O$ then we simply delete the nodes $x_{3}$ and $x_{4}$.

The simulation implemented by the subnetwork is, in this case, more involved. Let us assume that $w$ is a word that was sent towards the node $x$ in a communication step of $\Gamma$, and the word $w_{1} \#_{x} w_{2}$, with $w_{1} w_{2}=w$, was communicated in the network $\Gamma^{\prime}$. If the word $w$ can pass the input filters of $x$ then $w_{1} \#_{x} w_{2}$ can also enter $x_{0}$ (and no other node); the reversal holds as well. In the node $x$ we obtain from $w$ a word $w^{\prime}$ by substituting several $a$ symbols with $b$ symbols (actually, we either substitute exactly one occurrence of an $a$, if $a \notin F O$, all of them, if $a \in F O$, or none, if $w$ contains no $a$ ) and $w^{\prime}$ leaves the node if it verifies the output conditions. In the network $\Gamma^{\prime}$ the word is processed as follows. In $x_{0}$ it becomes $w_{1} \#_{x}^{\prime} w_{2}$ and is sent out. It can only enter $x_{1}$, if it contains at least on $a$, or $x_{2}$, otherwise. In the first case it becomes $w_{1}^{\prime} \#_{x}^{\prime} w_{2}^{\prime}$, by substituting exactly one $a$ symbol with a $b^{\prime}$ symbol. In the second case, the word becomes 
$w_{1} \#_{x}^{\prime \prime} w_{2}$ (only the symbol encoding the current node is changed, and the rest of the word remains the same because it contains no $a$ ). In the both cases, the obtained words enter $x_{5}$ where we obtain, in 5 processing steps, all the words $w_{3} \#_{x, y} w_{4}$, for a node $y$ such that $(x, y) \in E_{G}$ and $w_{3} w_{4}=w^{\prime}$, where $w^{\prime}$ was obtained from $w$ by substituting at most one $a$ symbol with a $b$ symbol. Such words can only be communicated $x_{6}$, if they $w^{\prime}$ verifies the output filters of $x$. In $x_{6}$ they are transformed into $w_{3} \#_{y} w_{4}$, and can go to the nodes of the subnetwork associated with the node $y$ of $\Gamma$. There is one more thing to be analyzed: the words that leave $x_{2}$ and contain $a$, in the case when $a \in F O$. These words can go to $x_{3}$, where they become $w_{1}^{\prime} \#_{x}^{\circ} w_{2}^{\prime}$ and, further, can only enter $x_{4}$ (but only if they do not contain any more forbidden output symbols of $x$, or their primed copies). In this node, $b^{\prime}$ becomes $b$ and $\#_{x}^{\circ}$ becomes $\#_{x}^{\prime}$, after 6 processing steps are made, and the obtained words are sent back to $x_{2}$, where another processing step of $x$ is simulated. It is not hard to see now that the action of the node $x$ in $\Gamma$ was correctly simulated by the subnetwork $s(x)$ in $\Gamma^{\prime}$; more precisely one processing and one communication step of $\Gamma$ are simulated in 9 processing and 9 communication steps of $\Gamma^{\prime}$.

The case of substitution nodes with weak filters can be treated in a similar fashion. We simply have to define the filters of $x_{0}$ and $x_{6}$ in the previous network as weak, and to set to $U^{\prime}$ their permitting output filters. As in the former case, if $w$ is a word that was sent towards the node $x$ in a communication step of $\Gamma$, and the word $w_{1} \#_{x} w_{2}$, with $w_{1} w_{2}=w$, was communicated in the network $\Gamma^{\prime}$, we obtain in $\Gamma^{\prime}$ all the words $w_{3} \#_{y} w_{4}$, such that $(x, y) \in E_{G}$ and $w_{3} w_{4}=w^{\prime}$ where $w^{\prime}$ was obtained in node $x$ from $w$ and was able to leave that node. Clearly, the action of the node $x$ in $\Gamma$ was simulated soundly by the subnetwork $s(x)$ in $\Gamma^{\prime}$.

Finally, we present the simulation of the deletion nodes. As in the case of insertions, we can have left, right or arbitrary deletion rules, and, strong or weak filters. However, all the cases are based on the same general idea. Therefore, we present and discuss in details only one case: the case of left deletion nodes, with strong filters. For right and arbitrary insertions we will describe only the differences from this construction.

Let us assume that the node $x$ verifies $\mathcal{N}(x)=(\{a \rightarrow \lambda\}, P I, F I, P O, F O), \alpha(x)=s$ and $\beta(x)=l$. First, we will assume that $a \in F O$. In this case, we have:

- $\operatorname{set}(s(x))=\left\{x_{0}, x_{1}, x_{2}, x_{3}, x_{5}\right\} \cup\left\{x_{b}, x^{b} \mid b \in U\right\}$.

- $\mathcal{N}\left(x_{0}\right)=\left(\left\{\lambda \rightarrow \#_{x}^{\prime}\right\}, P I, F I \cup\left(U_{1} \backslash\left\{\#_{x}\right\}\right), U^{\prime}, \emptyset\right), \alpha^{\prime}\left(x_{0}\right)=s, \beta^{\prime}\left(x_{0}\right)=r$.

- $\mathcal{N}\left(x_{1}\right)=\left(\left\{\#_{x} \rightarrow \lambda\right\},\left\{\#_{x}^{\prime}, \#_{x}\right\},\left(U_{1} \backslash\left\{\#_{x}, \#_{x}^{\prime}\right\}\right) \cup\left\{a^{\prime}\right\}, U^{\prime}, \emptyset\right), \alpha^{\prime}\left(x_{1}\right)=s, \beta^{\prime}\left(x_{1}\right)=*$.

- $\mathcal{N}\left(x_{2}\right)=\left(\left\{b \rightarrow b^{\prime} \mid b \in U\right\},\left\{\#_{x}^{\prime}\right\}, U_{1} \backslash\left\{\#_{x}^{\prime}\right\},\left\{b^{\prime} \mid b \in U\right\}, \emptyset\right), \alpha^{\prime}\left(x_{2}\right)=w, \beta^{\prime}\left(x_{2}\right)=*$.

- $\mathcal{N}\left(x_{3}\right)=\left(\left\{\#_{x}^{\prime} \rightarrow \#_{x}^{b} \mid b \in V\right\},\left\{b^{\prime} \mid b \in V\right\}, U_{1} \backslash\left\{\#_{x}^{\prime}\right\}, U^{\prime},\left\{\#_{x}^{\prime}\right\}\right), \alpha^{\prime}\left(x_{3}\right)=w, \beta^{\prime}\left(x_{3}\right)=*$.

- For all $b \in U$ we have: $\mathcal{N}\left(x_{b}\right)=\left(\left\{b^{\prime} \rightarrow \lambda\right\},\left\{\#_{x}^{b}\right\},\left\{c^{\prime} \mid c \in U \backslash\{b\}\right\} \cup\left\{c^{\prime \prime} \mid c \in U\right\}, U^{\prime},\left\{c^{\prime} \mid\right.\right.$ $c \in U\}), \alpha^{\prime}\left(x_{b}\right)=w, \beta^{\prime}\left(x_{b}\right)=l$.

- For all $b \in U \backslash\{a\}$ we have: $\mathcal{N}\left(x^{b}\right)=\left(\left\{\lambda \rightarrow b^{\prime \prime}\right\},\left\{\#_{x}^{b}\right\},\left\{c^{\prime} \mid c \in U\right\}, U^{\prime}, \emptyset\right), \alpha^{\prime}\left(x^{b}\right)=w$, $\beta^{\prime}\left(x^{b}\right)=l$.

- $\mathcal{N}\left(x^{a}\right)=\left(\left\{\#_{x}^{a} \rightarrow \#_{x}^{\prime \prime}\right\},\left\{\#_{x}^{a}\right\},\left\{c^{\prime} \mid c \in U\right\}, U^{\prime}, \emptyset\right), \alpha^{\prime}\left(x^{a}\right)=w, \beta^{\prime}\left(x^{a}\right)=*$.

- $\mathcal{N}\left(x_{4}\right)=\left(\left\{\#_{x}^{\prime \prime} \rightarrow \#^{(1)}, \#^{(1)} \rightarrow \#^{(2)}, \#^{(2)} \rightarrow \#^{(3)}, \#^{(3)} \rightarrow \#^{(4)}, \#^{(4)} \rightarrow \#_{x}^{\prime}\right\},\{a\},\left\{b^{\prime \prime} \mid b \in U\right\} \cup\right.$ $\left.\left(U_{1} \backslash\left\{\#_{x}^{\prime \prime}\right\}\right),\left\{\#_{x}^{\prime}\right\}, \emptyset\right), \alpha^{\prime}\left(x_{4}\right)=w, \beta^{\prime}\left(x_{4}\right)=*$.

- $\mathcal{N}\left(x_{5}\right)=\left(\left\{\#_{x}^{\prime \prime} \rightarrow \#^{(1)}, \#^{(1)} \rightarrow \#_{x, y}, \#_{x}^{b} \rightarrow \#_{x, y}, b^{\prime \prime} \rightarrow b \mid b \in U,(x, y) \in E_{G}\right\},\left\{\#_{x}^{\prime \prime}\right\} \cup\left\{b^{\prime \prime} \mid b \in\right.\right.$ $\left.U\}, F O \cup\left\{b^{\prime \prime} \mid b \in F O\right\} \cup\left(U_{1} \backslash\left\{\#_{x}^{\prime \prime}\right\}\right), U^{\prime},\left\{\#^{(1)}\right\} \cup\left\{\#_{x}^{\prime \prime}\right\} \cup\left\{b^{\prime \prime}, \#_{x}^{b} \mid b \in U\right\}\right), \alpha^{\prime}\left(x_{5}\right)=w$, $\beta^{\prime}\left(x_{5}\right)=*$. 
- $\mathcal{N}\left(x_{6}\right)=\left(\left\{\#_{x, y} \rightarrow \#_{y} \mid y \in X_{G}\right\}, P O,\left\{\#_{y}^{\prime \prime}, \#_{y}, \#_{y}^{\prime}, \#_{y}^{b} \mid b \in U, y \in X_{G}\right\}, \emptyset,\left\{\#_{x, y} \mid(x, y) \in\right.\right.$ $\left.\left.E_{G}\right\}\right), \alpha^{\prime}\left(x_{6}\right)=s, \beta^{\prime}\left(x_{6}\right)=*$.

The simulation implemented by the subnetwork is, in this case, more involved. Let us assume that $w$ is a word that was sent towards the node $x$ in a communication step of $\Gamma$, and the word $w_{1} \#_{x} w_{2}$, with $w_{1} w_{2}=w$, was communicated in the network $\Gamma^{\prime}$. If the word $w$ can pass the input filters of $x$ then $w_{1} \#_{x} w_{2}$ can also enter $x_{0}$, and vice versa. In the node $x$ we obtain from $w$ a word $w^{\prime}$ by deleting all the $a$ symbols from the left end of the word, and this word leaves the node if it verifies the output conditions. In the network $\Gamma^{\prime}$ the word is processed as follows. In $x_{0}$ it becomes $w_{1} \#_{x} w_{2} \#_{x}^{\prime}$ and is sent out. It can only enter $x_{1}$ where it becomes $w \#_{x}^{\prime}$. Now it can only go to $x_{2}$. Here it is transformed into $w_{1}^{\prime} b^{\prime} w_{2}^{\prime} \#_{x}^{\prime}$, for all $b \in U$ and $w_{1}^{\prime}, w_{2}^{\prime} \in U^{*}$ such that $w_{1}^{\prime} b w_{2}=w$. Now these word enter $x_{3}$, and the network obtains from them the words $w_{1}^{\prime} b^{\prime} w_{2}^{\prime} \#_{x}^{c}$, with $c \in U$. From these, only the words $w_{1}^{\prime} b^{\prime} w_{2}^{\prime} \#_{x}^{b}$ are further processed. More precisely, the node $x_{b}$ permits these words to enter, and transforms them into $w_{2}^{\prime} \#_{x}^{b}$, if and only if $w_{1}^{\prime}=\lambda$. Next, the obtained words can only go to node $x^{b}$. If $b \neq a$ it means that we simulated a deletion that should not have happened, so we remake the word into $b^{\prime \prime} w_{2}^{\prime} \#_{x}^{b}$; otherwise, the deletion was correct, and we get in $x^{a}$ the word $w_{2}^{\prime} \#_{x}^{\prime \prime}$. In the first case, the words can enter $x_{5}$ (if they do not contain any of the forbidden output symbols of node $x$ ) where they are transformed into $w^{\prime} \#_{x, y}$, with $(x, y) \in E_{G}$, in two processing steps, and sent out; finally, they can enter only the node $x_{6}$, but if and only if $w^{\prime}$ verifies the permitting output filters of $x$, and here are transformed into $w^{\prime} \#_{y}$ and go out in the network (and can only enter the nodes of the subnetwork constructed for the node $y$ ). In the second case, the word $w_{2}^{\prime} \#_{x}^{\prime \prime}$ can either enter $x_{5}$, and be processed as above, or, if it still contains $a$ symbols, which are forbidden output symbols for $x$, it goes to node $x_{4}$; in this node they are transformed into $w_{2}^{\prime} \#_{x}^{\prime}$ (in 5 steps), go back to node $x_{2}$, and the whole process described above is repeated. It is not hard to see now that the action of the node $x$ in $\Gamma$ was correctly simulated by the subnetwork $s(x)$ in $\Gamma^{\prime}$; more precisely one processing and one communication step of $\Gamma$ are simulated in 9 processing and 9 communication steps of $\Gamma^{\prime}$.

In the case when $a \notin F O$ we simply delete the node $x_{4}$, and the simulation goes on just the same. To adapt the above construction to the case of right deletion nodes, we should switch the working mode of nodes $x_{0}, x_{b}$ and $x^{b}$; that is, if one of these nodes was a node where left (right) operations were applied, it must become a node where right (respectively, left) operations are applied. In the case of arbitrary deletion nodes, we simply redefine all the node such that they apply the rules arbitrary. Finally, when the node $x$ has weak filters, we set the filters of $x_{1}$ and $x_{6}$ to weak and redefine their permitting output filters as equal to $U^{\prime}$, such that all the words that do not contain forbidden output symbols are allowed to leave these nodes.

The output node of the network is the node $O u t_{0}$ from the subnetwork $s(O u t)$, associated with the node Out. From the way the subnetworks $s(x)$, with $x \in X_{G}$, work we see that the following statements are equivalent:

i. $w$ is a word that entered the node $x$ in $\Gamma$, was transformed by this node into $w^{\prime}$ in one step, and $w^{\prime}$ was communicated to node $y$ (when $w^{\prime}$ cannot exit $x$ we assume that $y=x$ );

ii. the word $w_{1} \#_{x} w_{2}$, with $w_{1} w_{2}=w$, entered the node $x_{0}$, from the subnetwork $s(x)$ of $\Gamma^{\prime}$, and it was transformed, by the nodes of $s(x)$, into $w_{1}^{\prime} \#_{y} w_{2}^{\prime}$, with $w_{1}^{\prime} w_{2}^{\prime}=w^{\prime}$, in exactly 9 evolutionary and 9 communication steps.

Note that $\Gamma^{\prime}$ accepts a word $w$ if and only if a word $w_{1} \#_{O u t} w_{2}$, with $w_{1} w_{2} \in U^{*}$, can be derived from it; but such a word can be derived only in a number of steps divisible by 9 . 
According to the above, the computation of $\Gamma$ on $w$ ends (and accepts) in $t$ steps if and only if the computation of $\Gamma^{\prime}$ on $w$ ends (and, respectively, accepts) in $9 t$ steps. Therefore, $L(\Gamma)=L\left(\Gamma^{\prime}\right)$.

It is worth mentioning that the problem of simulating an arbitrary NEP by a complete NEP was approached also in the case of generating networks [4]. However, the things were different in that setting: the filters in GNEPs were regular sets, and this allowed us to control easier the communication; no weak/strong filtering conditions existed; each node contains a set of axioms, at the beginning of the computation. However, the most important differences are that: the rules were applied in a different fashion (i.e., if at least one rule can be applied to a word $w$, we obtain all the words that are derived from the word $w$ by applying exactly one of the possible rules at exactly one feasible position in the word $w$ ) and in the case of GNEPs we don't need to worry about halting computations, acceptance or rejection (and synchronizing the different derivations of a word, in order to obtain the same outcome).

Theorem 4.3 Given an ANEP $\Gamma=(V, U, \mu, G, \mathcal{N}, \alpha, \beta$, In, Out $)$, one can construct a complete ANEP $\Gamma^{\prime}=\left(V, U^{\prime}, \mu, G^{\prime}, \mathcal{N}^{\prime}, \alpha^{\prime}, \beta^{\prime}, I n^{\prime}, O u t^{\prime}\right)$ such that $\Gamma^{\prime}$ accepts (decides) the same language as $\Gamma$ accepts (respectively, decides). Moreover, two consecutive steps of $\Gamma$ (an evolutionary and a communication step) are simulated in exactly 54 consecutive steps (27 evolutionary and 27 communication steps) of $\Gamma^{\prime}$.

Proof. First, the given ANEP $\Gamma=\left(V, U, \mu, \nu, G, \mathcal{N}, \alpha, \beta, x_{I}, x_{O}\right)$, is transformed into an ANEP $\Gamma^{\prime}=\left(V, U, \mu, \nu, G^{\prime}, \mathcal{N}^{\prime}, \alpha^{\prime}, \beta^{\prime}, x_{I}^{\prime}, x_{O}^{\prime}\right)$ such that $\Gamma^{\prime}$ accepts (decides) the same language as $\Gamma$ accepts (respectively, decides), each node of $\Gamma^{\prime}$ has at most one rule and $x_{I}^{\prime}$ has no rules. Moreover, two consecutive steps of $\Gamma$ (an evolutionary and a communication step) are simulated in exactly in exactly 3 evolutionary steps and 3 communication steps of $\Gamma^{\prime}$. The proof of this fact can be found in [9].

Second, by Lemma 4.2, we transform $\Gamma^{\prime}$ into a complete ANEP $\Gamma^{\prime \prime}=\left(V, U^{\prime \prime}, \mu, \nu, G^{\prime \prime}, \mathcal{N}^{\prime \prime}\right.$, $\left.\alpha^{\prime \prime}, \beta^{\prime \prime}, x_{I}^{\prime \prime}, x_{O}^{\prime \prime}\right)$ such that $\Gamma^{\prime}$ accepts (decides) the same language as $\Gamma$ accepts (respectively, decides). Moreover, two consecutive steps of $\Gamma$ (an evolutionary and a communication step) are simulated in exactly in exactly 9 evolutionary steps and 9 communication steps of $\Gamma^{\prime}$.

This shows that the statements of Theorem 4.3 hold.

Compared to the afore mentioned simulation via Turing machines, we also propose a 2-steps construction, but the only computational model we use is the ANEP model. Also, the both steps rely on the same idea: we replace the nodes of the initial network with a group of nodes that simulate its job; this makes the construction simpler to apply, and easier to follow. Moreover, the complete network simulates in linear time the arbitrary network.

The result in Theorem 4.3 has a series of consequences. First, it provides a normal topology for ANEPs, allowing us to specify all the results and definitions in an uniform manner, without taking into account the particular topology of the network. Second, the transformation from an arbitrary network into a complete one is algorithmic, and can be applied automatically. Further, the number of nodes in the complete networks is greater only by a constant factor than the number of nodes of the simulated one. Our simulation preserves the computational properties of the initial ANEP, thus, complete networks can be used to prove lower bounds: the most time-efficient ANEP-based solution of a problem can be implemented on a complete ANEP.

The proof of Lemma 4.2 is an example on how one can design an ANEP by putting together 
multiple subnetworks; this approach seems close to that of procedural programming. Theorem 4.3 shows that building a greater ANEP from smaller subnetworks is an approach that can be used without being afraid that such a solution is no longer uniform (i.e., the network has very specific properties, and cannot be transformed efficiently to a general network, such as a complete one). Moreover, if an ANEP constructed from subnetworks works efficiently, so will do the complete variant of that ANEP.

\section{Computational Complexity}

We recall from [8] the following definition:

Definition 5.1 Let $M$ be a nondeterministic polynomial Turing machine and $w$ be a word over the input alphabet of $M$. The word $w$ is accepted by $M$ with respect to the shortest computations if one of the possible shortest computations of $M$ on $w$ is accepting; $w$ is rejected by $M$ w.r.t. the shortest computations if all the possible shortest computations of $M$ on $w$ are rejecting. We denote by $L_{s c}(M)$ the language decided by $M$ w.r.t. the shortest computations and by PTime sc $_{\text {. }}$ the class of all the languages decided in this manner.

It is not hard to see that the class of languages decided w.r.t. shortest computations by nonde-

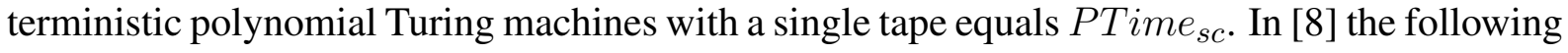
result is shown:

Theorem 5.2 PTime $\left._{s c}=\mathbf{P}^{\mathbf{N P}[\log ]} \cdot 1\right]$

Further, one can show that ANEPs simulate efficiently nondeterministic Turing machines that decide w.r.t. shortest computations, and vice versa.

\section{Theorem 5.3}

i. For every ANEP $\Gamma$, deciding a language $L$ and working in polynomial time $P(n)$, there exists a nondeterministic polynomial single-tape Turing machine $M$, deciding $L$ w.r.t. shortest computations; $M$ can be constructed such that it makes at most $P^{2}(n)$ steps in a halting computation on a string of length $n$.

ii. For every nondeterministic polynomial single-tape Turing machine $M$, deciding a language $L$ w.r.t. shortest computations, there exists a complete $A N E P \Gamma$, deciding the same language L. Moreover, $\Gamma$ can be constructed such that Time ${ }_{\Gamma}(n) \in \mathcal{O}(P(n))$, provided that $M$ makes at most $P(n)$ steps in a halting computation on a string of length $n$.

Proof. In order to define completely the setting, recall that a one-tape nondeterministic Turing machine is a construct $M=\left(Q, V, U, \delta, q_{0}, B, F\right)$, where $Q$ is a finite set of states, $V$ is the input alphabet, $U$ is the tape alphabet, $V \subset U, q_{0}$ is the initial state, $B \in U \backslash V$ is the "blank" symbol, $F \subseteq Q$ is the set of final states, and $\delta$ is the transition mapping, $\delta:(Q \backslash F) \times U \rightarrow$ $2^{Q \times(U \backslash\{B\}) \times\{R, L\}}$. In this paper, we assume without loss of generality that any Turing machine we consider has a semi-infinite tape (bounded to the left) and makes no stationary moves; the computation of such a machine is described in [14, 5, 13]. We are interested here in machines

\footnotetext{
${ }^{1} \mathbf{P}^{\mathbf{N P}[\log ]}$ is the class of problems solvable by a deterministic polynomial machine, that can make $\mathcal{O}(\log n)$ queries to an NP oracle (where $n$ is the length of the input).
} 
that accept a language w.r.t. shortest computations. The word $w$ is accepted by $M$ with respect to the shortest computations if one of the possible shortest computations of $M$ on $w$ is accepting; $w$ is rejected by $M$ w.r.t. the shortest computations if all the possible shortest computations of $M$ on $w$ are rejecting. We denote by $L_{s c}^{a}(M)$ the accepted language, i.e., the set of all the words accepted by $M$; if all the computations of $M$ are either accepting or rejecting, we say that $M$ decides the accepted language, and denote by $L_{s c}(M)$ the language decided by $M$ w.r.t. the shortest computations.

First we show that $\mathbf{i}$. holds. The idea is quite simple. Since $\Gamma$ works in polynomial time it follows that any computation of this ANEP on a word of length $n$ has at most $P(n)$ steps. A nondeterministic Turing machine chooses nondeterministically a computation and simulates it, exactly like in the proofs from [10]. The only restriction is that the machine first computes $n+P(n)$ and, then, will always simulate one evolutionary step, as well as one communication step, in exactly $n+P(n)$ computational steps. A computation halts when the word obtained can be accepted in the output node of $\Gamma$, and it accepts when the word contains the special accepting symbol, and rejects, otherwise. In this way, the shortest computation of the Turing machine on an input word $w$ simulates the shortest possible derivation of $\Gamma$ starting from that word and ending with a word accepted in the output node. The computation of the machine accepts (rejects), w.r.t. shortest computations, if and only the computation of the ANEP accepts (rejects). Clearly, $M$ makes at most $n P(n)+P^{2}(n)$ steps in a halting computation. This concludes the proof of $\mathbf{i}$.

Now we move on to show ii.. We start by constructing an incomplete network $\Gamma^{\prime}$ that simulates $M$. Then we just have to apply Theorem 4.3 and we obtain the desired result.

We assume, with out losing generality, that $M$ has exactly one accepting state $q_{o}$ and a blocking state (i.e., a state in which it enters and rejects) $q_{r}$. We assume that there are no transitions that can occur in these states.

The main idea is to construct $\Gamma^{\prime}$ from several subnetworks. The working alphabet of $\Gamma^{\prime}$ is denoted by $U_{\Gamma^{\prime}}$.

First we have two nodes $x_{1}$ and $x_{2}$ that work as follows.

- $\mathcal{N}\left(x_{1}\right)=(\{\lambda \rightarrow B\}, \emptyset, \emptyset, U, \emptyset), \alpha\left(x_{1}\right)=w, \beta\left(x_{1}\right)=r$.

- $\mathcal{N}\left(x_{2}\right)=\left(\left\{\lambda \rightarrow q_{0}\right\}, U, \emptyset, U \cup\left\{q_{0}\right\}, \emptyset\right), \alpha\left(x_{2}\right)=w, \beta\left(x_{2}\right)=r$.

- We have the edge $\left(x_{1}, x_{2}\right)$

Then we have a node $x_{\text {center }}$, which acts as a central node of the network, as it basically controls the way the computation is conducted.

- $\mathcal{N}\left(x_{\text {center }}\right)=\left(\left\{q \rightarrow\left[q, a, q^{\prime}, b, X\right], q_{o} \rightarrow q_{o}, q_{r} \rightarrow q_{r} \mid q, q^{\prime} \in Q, a, b \in U, X \in\{R, L\}\right.\right.$, and $\left.\left.\left(q^{\prime}, b, X\right) \in \delta(q, a)\right\}, U \cup Q, \emptyset, U_{\Gamma^{\prime}}, Q\right), \alpha\left(x_{\text {center }}\right)=w, \beta\left(x_{\text {center }}\right)=r$.

- We have the edge $\left(x_{2}, x_{\text {center }}\right)$.

We also have an output node:

- $\mathcal{N}\left(x_{\text {out }}\right)=\left(\emptyset,\left\{q_{a}, q_{b}\right\}, \emptyset, \emptyset, \emptyset\right), \alpha\left(x_{\text {center }}\right)=w$.

- We have the edge $\left(x_{\text {center }}, x_{\text {out }}\right)$.

For a transition $\left(q^{\prime}, b, L\right) \in \delta(q, B)$, let $t$ denote the tuple $\left(q, B, q^{\prime}, b, L\right)$. We have a subnetwork $\Gamma_{t}^{\prime}$. This subnetwork has the following nodes and edges: 
- $\mathcal{N}\left(x_{1}^{t}\right)=\left(\{B \rightarrow \perp\},\{B\}, Q \cup\left(Q^{\prime} \backslash\left\{\left[q, B, q^{\prime}, b, L\right]\right)\right\},\{\perp\}, \emptyset\right), \alpha\left(x_{1}^{t}\right)=w$.

- $\mathcal{N}\left(x_{2}^{t}\right)=\left(\{\perp \rightarrow \lambda\},\{\perp\}, \emptyset, U_{\Gamma^{\prime}}, \emptyset\right), \alpha\left(x_{2}^{t}\right)=w, \beta\left(x_{2}^{t}\right)=l$.

- $\mathcal{N}\left(x_{3}^{t}\right)=\left(\{\lambda \rightarrow B\}, U_{\Gamma^{\prime}},\{\perp\}, U_{\Gamma^{\prime}}, \emptyset\right), \alpha\left(x_{3}^{t}\right)=w, \beta\left(x_{3}^{t}\right)=l$.

- $\mathcal{N}\left(x_{4}^{t}\right)=\left(\{\lambda \rightarrow b\}, U_{\Gamma^{\prime}}, \emptyset, U_{\Gamma^{\prime}}, \emptyset\right), \alpha\left(x_{4}^{t}\right)=w, \beta\left(x_{4}^{t}\right)=l$.

- $\mathcal{N}\left(x_{5}^{t}\right)=\left(\left\{c \rightarrow c^{\prime} \mid c \in U\right\}, U_{\Gamma^{\prime}}, \emptyset,\left\{c^{\prime} \mid c \in U\right\}, \emptyset\right), \alpha\left(x_{5}^{t}\right)=w$.

- $\mathcal{N}\left(x_{c, 1}^{t}\right)=\left(\{\lambda \rightarrow c\},\left\{c^{\prime}\right\}, \emptyset, U_{\Gamma^{\prime}}, \emptyset\right), \alpha\left(x_{c, 1}^{t}\right)=w, \beta\left(x_{c, 1}^{t}\right)=l$, for $c \in U \backslash\{B\}$.

- $\mathcal{N}\left(x_{6}^{t}\right)=\left(\left\{c^{\prime} \rightarrow \lambda \mid c \in U\right\},\left\{c^{\prime} \mid c \in U\right\}, \emptyset, U_{\Gamma^{\prime}}, \emptyset\right), \alpha\left(x_{6}^{t}\right)=w, \beta\left(x_{6}^{t}\right)=r$.

- $\mathcal{N}\left(x_{7}^{t}\right)=\left(\left\{\left[q, B, q^{\prime}, b, L\right] \rightarrow q^{\prime}\right\}, U_{\Gamma^{\prime}},\left\{c^{\prime} \mid c \in U\right\}, Q, \emptyset\right), \alpha\left(x_{7}^{t}\right)=w$.

- We have the edges $\left(x_{i}^{t}, x_{i+1}^{t}\right)$, for $i \in\{1,2,3,4,6\}$, and the edges $\left\{\left(x_{5}^{t}, x_{c}^{t}\right),\left(x_{c}^{t}, x_{6}^{t}\right) \mid c \in\right.$ $U \backslash\{B\}\}$. Furthermore, we have the edges $\left(x_{\text {center }}, x_{1}^{t}\right)$ and $\left(x_{7}^{t}, x_{\text {center }}\right)$

For a transition $\left(q^{\prime}, b, R\right) \in \delta(q, B)$, let $t$ denote the tuple $\left(q, B, q^{\prime}, b, R\right)$. We have a subnetwork $\Gamma_{t}^{\prime}$. This subnetwork has the following nodes and edges:

- $\mathcal{N}\left(x_{1}^{t}\right)=\left(\{B \rightarrow \perp\},\{B\}, Q \cup\left(Q^{\prime} \backslash\left\{\left[q, B, q^{\prime}, b, L\right]\right)\right\},\{\perp\}, \emptyset\right), \alpha\left(x_{1}^{t}\right)=w$.

- $\mathcal{N}\left(x_{2}^{t}\right)=\left(\{\perp \rightarrow \lambda\},\{\perp\}, \emptyset, U_{\Gamma^{\prime}}, \emptyset\right), \alpha\left(x_{2}^{t}\right)=w, \beta\left(x_{2}^{t}\right)=l$.

- $\mathcal{N}\left(x_{3}^{t}\right)=\left(\{\lambda \rightarrow B\}, U_{\Gamma^{\prime}},\{\perp\}, U_{\Gamma^{\prime}}, \emptyset\right), \alpha\left(x_{3}^{t}\right)=w, \beta\left(x_{3}^{t}\right)=l$.

- $\mathcal{N}\left(x_{4}^{t}\right)=\left(\{\lambda \rightarrow b\}, U_{\Gamma^{\prime}}, \emptyset, U_{\Gamma^{\prime}}, \emptyset\right), \alpha\left(x_{4}^{t}\right)=w, \beta\left(x_{4}^{t}\right)=r$.

- $\mathcal{N}\left(x_{5}^{t}\right)=\left(\left\{\left[q, a, q^{\prime}, b, L\right] \rightarrow \perp_{1}, \perp_{1} \rightarrow \perp_{2}, \perp_{2} \rightarrow \perp_{3}, \perp_{3} \rightarrow q^{\prime}\right\}, U_{\Gamma^{\prime}},\left\{c^{\prime} \mid c \in U\right\}, Q,\left\{\perp_{i} \mid i \in\right.\right.$ $\{1,2,3\}\}), \alpha\left(x_{5}^{t}\right)=w$.

- We have the edges $\left(x_{i}^{t}, x_{i+1}^{t}\right)$, for $i \in\{1,2,3,4\}$. Also, we have the edges $\left(x_{c e n t e r}, x_{1}^{t}\right)$ and $\left(x_{4}^{t}, x_{\text {center }}\right)$

For a transition $\left(q^{\prime}, b, L\right) \in \delta(q, a)$, with $a \neq B$, let $t$ denote the tuple $\left(q, a, q^{\prime}, b, L\right)$. We have a subnetwork $\Gamma_{t}^{\prime}$. This subnetwork has the following nodes and edges:

- $\mathcal{N}\left(x_{1}^{t}\right)=\left(\{a \rightarrow \perp\},\{a\}, Q \cup\left(Q^{\prime} \backslash\left\{\left[q, B, q^{\prime}, b, L\right]\right)\right\},\{\perp\}, \emptyset\right), \alpha\left(x_{1}^{t}\right)=w$.

- $\mathcal{N}\left(x_{2}^{t}\right)=\left(\{\perp \rightarrow \lambda\},\{\perp\}, \emptyset, U_{\Gamma^{\prime}}, \emptyset\right), \alpha\left(x_{2}^{t}\right)=w, \beta\left(x_{2}^{t}\right)=l$.

- $\mathcal{N}\left(x_{3}^{t}\right)=\left(\{\lambda \rightarrow b\}, U_{\Gamma^{\prime}},\{\perp\}, U_{\Gamma^{\prime}}, \emptyset\right), \alpha\left(x_{3}^{t}\right)=w, \beta\left(x_{3}^{t}\right)=l$.

- $\mathcal{N}\left(x_{4}^{t}\right)=\left(\left\{c \rightarrow c^{\prime} \mid c \in U\right\}, U_{\Gamma^{\prime}}, \emptyset,\left\{c^{\prime} \mid c \in U\right\}, \emptyset\right), \alpha\left(x_{5}^{t}\right)=w$.

- $\mathcal{N}\left(x_{c, 1}^{t}\right)=\left(\{\lambda \rightarrow c\},\left\{c^{\prime}\right\}, \emptyset, U_{\Gamma^{\prime}}, \emptyset\right), \alpha\left(x_{c, 1}^{t}\right)=w, \beta\left(x_{c, 1}^{t}\right)=l$, for $c \in U \backslash\{B\}$.

- $\mathcal{N}\left(x_{5}^{t}\right)=\left(\left\{c^{\prime} \rightarrow \lambda \mid c \in U\right\},\left\{c^{\prime} \mid c \in U\right\}, \emptyset, U_{\Gamma^{\prime}}, \emptyset\right), \alpha\left(x_{6}^{t}\right)=w, \beta\left(x_{6}^{t}\right)=r$, for $c \in U \backslash\{B\}$.

- $\mathcal{N}\left(x_{6}^{t}\right)=\left(\left\{\left[q, a, q^{\prime}, b, L\right] \rightarrow \perp, \perp \rightarrow q^{\prime}\right\}, U_{\Gamma^{\prime}},\left\{c^{\prime} \mid c \in U\right\}, Q,\{\perp\}\right), \alpha\left(x_{7}^{t}\right)=w$.

- We have the edges $\left(x_{i}^{t}, x_{i+1}^{t}\right)$, for $i \in\{1,2,3,4,5\}$, and the edges $\left\{\left(x_{4}^{t}, x_{c}^{t}\right),\left(x_{c}^{t}, x_{5}^{t}\right) \mid c \in\right.$ $U \backslash\{B\}\}$. Furthermore, we have the edges $\left(x_{\text {center }}, x_{1}^{t}\right)$ and $\left(x_{6}^{t}, x_{\text {center }}\right)$

For a transition $\left(q^{\prime}, b, R\right) \in \delta(q, a)$, with $a \neq B$, let $t$ denote the tuple $\left(q, a, q^{\prime}, b, R\right)$. We have a subnetwork $\Gamma_{t}^{\prime}$. This subnetwork has the following nodes and edges:

- $\mathcal{N}\left(x_{1}^{t}\right)=\left(\{a \rightarrow \perp\},\{a\}, Q \cup\left(Q^{\prime} \backslash\left\{\left[q, B, q^{\prime}, b, L\right]\right)\right\},\{\perp\}, \emptyset\right), \alpha\left(x_{1}^{t}\right)=w$.

- $\mathcal{N}\left(x_{2}^{t}\right)=\left(\{\perp \rightarrow \lambda\},\{\perp\}, \emptyset, U_{\Gamma^{\prime}}, \emptyset\right), \alpha\left(x_{2}^{t}\right)=w, \beta\left(x_{2}^{t}\right)=l$. 
- $\mathcal{N}\left(x_{3}^{t}\right)=\left(\{\lambda \rightarrow b\}, U_{\Gamma^{\prime}},\{\perp\}, U_{\Gamma^{\prime}}, \emptyset\right), \alpha\left(x_{3}^{t}\right)=w, \beta\left(x_{3}^{t}\right)=r$.

- $\mathcal{N}\left(x_{4}^{t}\right)=\left(\left\{\left[q, a, q^{\prime}, b, L\right] \rightarrow \perp_{1}, \perp_{4} \rightarrow q^{\prime}\right\} \cup\left\{\perp_{i} \rightarrow \perp_{i+1} \mid 1 \leq i \leq 3\right\}, U_{\Gamma^{\prime}},\left\{c^{\prime} \mid c \in U\right\}, Q\right.$, $\left.\left\{\perp_{i} \mid i \in\{1,2,3,4\}\right\}\right), \alpha\left(x_{4}^{t}\right)=w$.

- We have the edges $\left(x_{i}^{t}, x_{i+1}^{t}\right)$, for $i \in\{1,2,3\}$. Furthermore, we have the edges $\left(x_{c e n t e r}, x_{1}^{t}\right)$ and $\left(x_{3}^{t}, x_{c e n t e r}\right)$

The way the network $\Gamma^{\prime}$ works is quite simple: the nodes $x_{1}$ and $x_{2}$ transform the input word $w$ into $w B q_{0}$, and sends this word to $x_{c e n t e r}$. Then, we can assume that the node $x_{c e n t e r}$ contains a word $w^{\prime} B q w^{\prime \prime}$, meaning that the machine $M$ is in state $q$ and the tape-head points to the first symbol of $w^{\prime} B$ (assumption that holds after the first step). This node chooses which transition of $M$ should be simulated next. Let us assume that the machine chooses to simulate a move given by the transition $(q, b, L) \in \delta(q, B)$. In this case, the word becomes $w^{\prime} B\left[q, B, q^{\prime}, b, L\right] w^{\prime \prime}$ and is sent to the subnetwork $\Gamma_{t}^{\prime}$. Here, in the nodes $x_{1}^{t}$ and $x_{2}^{t}$ we check if $B$ is the leftmost symbol, and it is deleted; if $B$ was not the leftmost symbol, the word is blocked, and this derivation stops. Then, in the nodes $x_{3}^{t}$ and $x_{4}^{t}$ another $B$ is inserted (the machine-tape contains an infinite number of $B \mathrm{~s}$, but we always keep just the first one in our words, and, in this case, this $B$ was deleted), and the $b$ symbol that replaced the deleted $B$ is inserted at the leftmost end of the word. Now we should simulate the movement of the head, that is we should move one symbol from the rightmost end to the leftmost end. And this is done in the nodes $x_{5}^{t}, x_{c}^{t}$ and $x_{6}^{t}$. The word first becomes $b B\left[q, B, q^{\prime}, a L\right] x c^{\prime} y$, where $x c y=w^{\prime \prime}$, and, then, $c b B\left[q, B, q^{\prime}, b, L\right] x c^{\prime} y$. Now $c^{\prime}$ is deleted if and only if it is the rightmost symbol, and we obtain the word $c b B\left[q, B, q^{\prime}, b, L\right] x$; otherwise, the word is lost. Finally, in $x_{7}^{t}$ the word becomes $c b B q^{\prime} x$, and it is sent back to $x_{c e n t e r}$, where a new move of the machine will be simulated.

The other types of moves are simulated in a very similar manner (actually, the case described above was the most involve one). Note that each move is simulated in exactly 9 evolutionary and 9 communication steps by the ANEP: 1 evolutionary and 1 communication step for choosing which move is simulated, and, then, 8 evolutionary and 8 communication steps for simulating the move. In some of the cases, we needed to do some extra dummy-moves, just to be able to synchronize all the possible derivations.

The node $x_{\text {center }}$ can also leave the word unchanged, if it contains $q_{a}$ or $q_{b}$. Such words go to $x_{\text {out }}$. The special accepting symbol of the network is $q_{a}$.

It is not hard to see that a halting computation of $M$, with $t$ steps, is simulated by $\Gamma^{\prime}$ in $6+18 t$ consecutive steps, and it ends with a word in the node $x_{\text {out }}$. Also, the only words that can enter in the output node of $\Gamma^{\prime}$ have the form $w^{\prime} B q_{c} w^{\prime \prime}$, with $c \in\{a, b\}$, provided that the configuration $w^{\prime \prime} q_{c} w^{\prime} B$ can be reached by $M$. Also, such a word enters in that node only after $6+18 k$ steps, with $k \in I N$. So the words that will be accepted the first in the node $x_{\text {out }}$ are exactly those encoding the final configurations reached in the shortest computations of $M$.

Therefore, the words that are accepted (respectively, rejected) by $\Gamma^{\prime}$ are exactly those that are accepted (respectively, rejected) by $M$, w.r.t. shortest computations.

As a consequence of Theorem 5.3 we obtain the following Theorem.

Theorem 5.4 PTime PNEP $=\mathbf{P}^{\mathrm{NP}[\log ]}$.

This result seems interesting to us as we are not aware of any other characterization of the class $\mathbf{P}^{\mathbf{N P}[\log ]}$ by computational complexity classes defined for bio-inspired computing models. Note, 
once more, that all the problems in $\mathbf{P}^{\mathbf{N P}[\log ]}$ can be solved by networks with the same topology, so, in a way, the solution to a problem is not based on the topology of the network but on the algorithm implemented by that network.

Finally, we show that, in fact, one can design complete ANEPs working faster than nondeterministic Turing machines. This shows that solving a problem by nondeterministic Turing machines and then simulate such machines by NEPs does not lead to an optimal ANEP-based solution to that problem. Also, this shows that the mechanism implemented by ANEPs does more than replacing the nondeterminism by massive parallelism.

Example 5.5 Let $L=\left\{a^{n} b \mid n \in I N, n \geq 1\right\}$. It is not hard to see that any nondeterministic Turing machine, deciding $L$ in the classical way, w.r.t. shortest computations or using oracles, and with an arbitrary number of tapes, makes at least a linear number of moves before it stops on an input word. However, $L$ can be accepted in constant time by a complete ANEP.

- We construct an ANEP $\Gamma$ with an underlying graph with 6 nodes $x_{1}, x_{2}, x_{3}, x_{4}, x_{5}$, and $x_{\text {out }}$, and edges $\left(x_{1}, x_{2}\right),\left(x_{1}, x_{4}\right),\left(x_{2}, x_{3}\right),\left(x_{2}, x_{5}\right),\left(x_{3}, x_{\text {out }}\right),\left(x_{4}, x_{5}\right),\left(x_{5}, x_{\text {out }}\right)$.

- This ANEP works as follows: in the node $x_{1}$ (which is also the input node) a $b$ symbol from the input word is replaced with an $X$, and all the words obtained exist the node. They can go to $x_{2}$, where they enter only if they have an $X$ (so only words that initially contained at least a $b$ symbol can be further processed in this node); all the other words go to $x_{4}$. In $x_{2}$ the rightmost symbol of any words deleted if and only if it is an $X$. Further, the words can go to $x_{3}$, if they have no $X$ and still have an $a$, or to $x_{5}$, otherwise. Clearly, at least a word enters $x_{3}$ if and only if the input word had the form $a^{n} b$, with $n \in I N, n \geq 1$. In this node a special $\mu$ symbol is inserted in the words. The nodes $x_{4}$ and $x_{5}$ do not process the words in any way. All the words obtained in $x_{3}$ and $x_{5}$ go to $x_{\text {out }}$, and the input word is accepted if and only if one of the words in $x_{\text {out }}$ contains $\mu$.

- From the explanations above it follows clearly that $\Gamma$ accepts $L$, and any word is decided in at most 3 evolutionary steps and communication steps.

- By Theorem 4.3 we obtain that there exists a complete network $\Gamma^{\prime}$ accepting $L$ in constant time.

\section{References}

[1] A. Alhazov, E. Csuhaj-Varjú, C. Martín-Vide, Y. Rogozhin, On the size of computationally complete hybrid networks of evolutionary processors. Theor. Comput. Sci. 410 (2009) 35, 3188-3197.

[2] P. Bottoni, A. Labella, F. Manea, V. Mitrana, J. M. Sempere, Filter Position in Networks of Evolutionary Processors Does Not Matter: A Direct Proof. In: DNA Computing and Molecular Programming: 15th International Conference, DNA 15, Revised Selected Papers, LNCS 5877. Springer-Verlag, Berlin, Heidelberg, 2009, 1-11.

[3] E. Csuhaj-Varjú, A. SalomaA, Networks of Parallel Language Processors. In: New Trends in Formal Languages - Control, Cooperation, and Combinatorics (to Jürgen Dassow on the occasion of his 50th birthday), LNCS 1218. Springer-Verlag, London, UK, 1997, 299-318. 
[4] J. Dassow, F. Manea, B. TRuthe, A Normal Form for Networks of Evolutionary Processors. Technical report, Otto-von-Guericke-Universität Magdeburg, Fakultät für Informatik, 2010. Http://theo.cs.uni-magdeburg.de/pubs/preprints/pp-afl-2010-02.pdf.

[5] J. Hartmanis, R. E. Stearns, On the Computational Complexity of Algorithms. Trans. Amer. Math. Soc. 117 (1965), 533-546.

[6] W. D. Hillis, The Connection Machine. MIT Press, Cambridge, MA, USA, 1986.

[7] R. Loos, F. Manea, V. Mitrana, Small Universal Accepting Hybrid Networks of Evolutionary Processors. Acta Inf. 47 (2010) 2, 133-146.

[8] F. MANEA, Deciding according to the shortest computations. Technical report, Ottovon-Guericke-Universität Magdeburg, Fakultät für Informatik, 2010. Http://theo.cs.unimagdeburg.de/pubs/preprints/pp-afl-2011-02.pdf.

[9] F. ManeA, Deciding Networks of Evolutionary Processors. Technical report, Ottovon-Guericke-Universität Magdeburg, Fakultät für Informatik, 2010. Http://theo.cs.unimagdeburg.de/pubs/preprints/pp-afl-2011-05.pdf.

[10] F. Manea, M. Margenstern, V. Mitrana, M. J. Perez-Jimenez, A New Characterization of NP, P and PSPACE with Accepting Hybrid Networks of Evolutionary Processors. Theor. Comp. Sys. 46 (2010) 2, 174-192.

[11] F. Manea, C. Martín-Vide, V. Mitrana, Accepting Networks of Evolutionary Word and Picture Processors: A Survey. In: C. MARTÍn-Vide (ed.), Scientific Applications of Language Methods. Mathematics, Computing, Language, and Life: Frontiers in Mathematical Linguistics and Language Theory 2, World Scientific, 2010, 525-560.

[12] M. Margenstern, V. Mitrana, M. J. PéreZ-Jiménez, Accepting Hybrid Networks of Evolutionary Processors. In: Proc. DNA 2004, LNCS 3384. 2004, 235-246.

[13] C. M. Papadimitriou, Computational Complexity. Addison-Wesley, Reading, Massachusetts, 1994.

[14] G. Rozenberg, A. SAlomaA, Handbook of Formal Languages. Springer-Verlag New York, Inc., Secaucus, NJ, USA, 1997. 